\title{
Development and operational experience of the web based application to collect, manage, and release the alignment and calibration configurations for data processing at CMS
}

\author{
Hasbuddin $\mathrm{Md}^{1, *}$, Arun $\mathrm{Kumar}^{2}$, Giacomo Govi ${ }^{3}$, Giovanni Franzoni ${ }^{4}$, Luca Pernie ${ }^{5,}$, and \\ Sverre Herland ${ }^{6}$, \\ ${ }^{1}$ Department of Physics and Astrophysics, University of Delhi, India \\ ${ }^{2}$ Rice University, Houston, USA \\ ${ }^{3}$ Fermi National Accelerator Laboratory, Batavia, USA \\ ${ }^{4}$ CERN, Geneva, Switzerland \\ ${ }^{5}$ Texas A\&M University, USA \\ ${ }^{6}$ Norwegian University of Science and Technology, Norway
}

\begin{abstract}
.
The alignment and calibration workflows at the Compact Muon Solenoid (CMS) experiment are fundamental to provide a high quality physics data and to maintain the design performance of the experiment. To facilitate the operational efforts required by the experiment, the alignment and calibration team has developed and deployed a set of web-based applications to search, navigate and prepare a consistent set of calibrations to be consumed in reconstruction of data for physics, accessible through the Condition DB Browser. The Condition DB Browser hosts also various data management tools, including a visualization tool that allows to easily inspect alignment an calibration contents, an user-defined notification agent for delivering updates on modification to the database, a logging service for the user and the automatic online-to-offline condition uploads. In this paper we report on the operational experience of this web application from 2017 data taking, with focus on new features and tools incorporated during this period.
\end{abstract}

\section{Introduction}

The CMS is a multipurpose detector operated at the CERN Large Hadron Collider (LHC)[1]. The primary goal of the CMS experiment is to explore physics at the TeV energy scale. The CMS experiment features a two-level trigger architecture whose purpose is to reduce the event rate. The first Level (L1) Trigger is implemented in hardware and the second High Level Trigger (HLT)[2] in software. Calibration and alignment data are fundamental to maintain the design performance of the experiment. Dedicated workflows have been put in place to compute and validate the alignment and calibration sets and insert them in the conditions database before the (re-construction)[2] process starts. There are several different workflows at CMS which consume non-event data:

- HLT to select the collisions to be recorded

\footnotetext{
*e-mail: hasib.md@cern.ch
} 
- The processing of the data of recorded collisions

- The production of Monte Carlo simulated events, used for comparison with data, or for studies of detector upgrades

\section{Conditions Data in CMS experiments}

\subsection{Conditions Overview}

While event data contain information about physics collisions, alignment and calibration conditions are non-event data which describe the evolving status and performance of the several detector components of CMS. Conditions are stored in relational databases as a set of binary objects (BLOBs)[3], serialised using boost libraries within the CMS offline software framework. The population of condition databases with calibration constants must be fast and reliable, since these data are not only used in the offline reconstruction chain and for physics analysis, but also in the online data-taking algorithms. A dedicated service allows experts to update the alignment and calibration sets on the master production database, and provides immediate feedback on the outcome of the upload.

\subsection{Conditions data model}

A proper reconstruction of collision events makes use of non-event data (the so-called condition data), such as alignment and calibration constants, that are stored in the Oracle database and are accessed during the execution of reconstruction applications. The condition data include data from any detector subsystem describing its state and constants of calibrations; they are mainly determined offline. Conditions data (payloads) are accompanied by tags (specifying the subsystem and the payload version) and IOVs (the temporal range over which the data is valid).

Payload The "atom" of every conditions is called the Payload. It represents the set of parameters consumed in the workflows of the event data processing. It is associated to a user-defined $\mathrm{C}++$ class in CMS software categorised as CondFormat (Payload Type)[4], each CondFormat is specific to one detector and contains alignment and calibration variables for it. The payload data is exchanged and stored as an unstructured binary array, with no assumption on its internal layout.

Record In the CMS software framework, a dedicated C++ class (the Record) acts as entry point for a Payload by importing the database content during CMS software Run time. Every processing workflow for event data involves a specific set of Records, each of which required valid conditions.

Interval Of Validity (IOV) is the time interval during which the constants held by a Payload apply. In this context, time is represented by a Run number (identifier of the event data set collected in a given time span), luminosity section value, or an universal timestamp.

Tag A fully defined sequence of conditions comprising a set of Payloads and their associate IOVs covering the time span required by the workload.

Global Tag is a complete set of Tags assigned to the Records involved in a given workflow. Because of that the production managers can handle specific collections of Tags and configure various jobs to access different subsets of them for large-scale data production. 


\section{Overview of the CMS Conditions Browser}

The CMS conditions database browser is a web service (cmsDbBrowser)[5] which was created to streamline and structure the management of alignment and calibration. A complete alignment and calibration scenario is factored in ( 200$)$ of records, which are updated independently and can have a time-dependent content, to reflect the evolution of the detector and data taking conditions. Given the complexity of the CMS condition scenarios and the large number of experts $(\sim 50)$ who actively measure and release calibration data, in 2015 a novel web-based service was developed to structure and streamline their management. It does so by:

1. Providing an intuitive and easy way to inspect, navigate and search the existing conditions data and metadata to any CMS member;

2. Easing the bookkeeping and management of the condition metadata by condition managers;

3. Handling the workflow for the update requests of the Global Tags, submitted by detector expert;

4. Providing monitoring information for tracing the changes in the Condition data used for the production workflows.

\subsection{Service Architecture}

The CmsDbBrowser was designed to work with condition data, using the Oracle read-only Active Data Guard(ADG) copy which stores replicated content of the master condition database. For security and reliability reasons, calibration data can be written in the condition database only from within the technical network of the experiment which is protected by a firewall separating it from the general purpose CERN network.

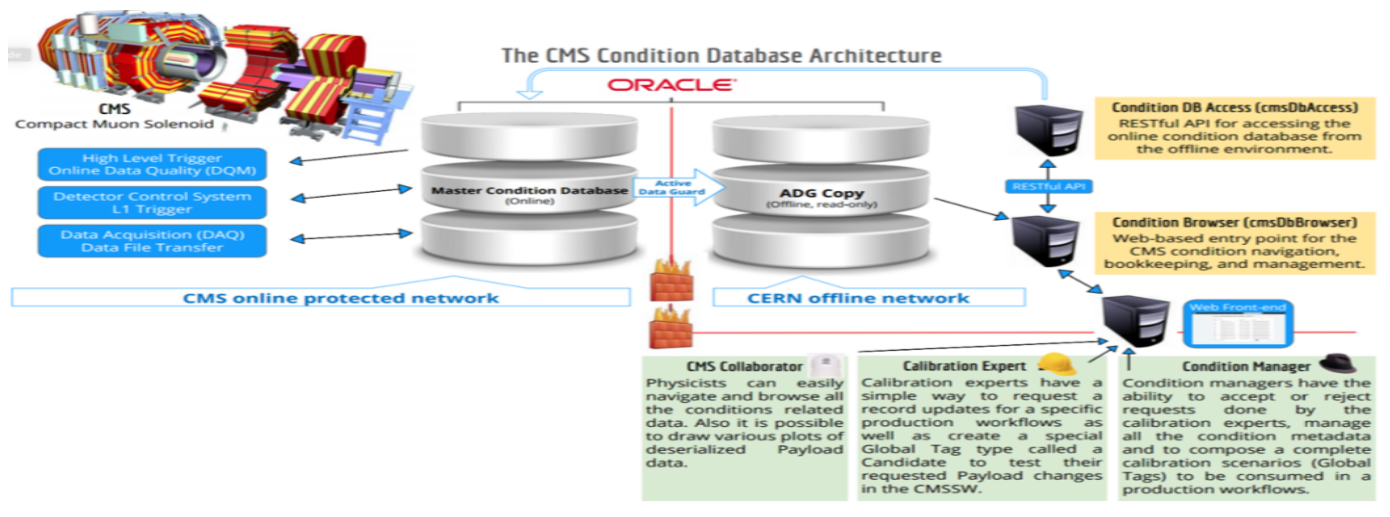

Figure 1. The CMS Condition Browser Service Architecture

As shown in figure 1, this design does not allow a direct export to the master Oracle database where all the conditions data are stored. To solve these issues, a RESTful API to the main Condition DB was created as a separate service called cmsDbAccess. In this way the access to the DB is separated and it makes cmsDbBrowser isolated and more secure. The cmsDbAccess API is deployed in the special machine which has an open gateway to the CMS technical environment and can access the main condition database directly. 


\subsection{Design and Implementation choices}

The Browser is able to search a user keyword in all the metadata. The result of the search is presented in a categorized way per Tag, Global Tag and Payloads. It is also possible to list the content of the Tag, Global Tag and Payloads selected in query results. The backend of cmsDbBrowser is implemented in Python programming language using the Flask web framework. An Object Relational Mapper (ORM) SQLAlchemy is used which handles all the database transactions. For the frontend the Bootstrap CSS framework is used together with the jQuery and Highcharts JavaScript libraries, which allow the creation of a modern user interface with advanced functionality. This set of technologies have proven to be reliable, efficient and secure. It meets our end-users requirements and provides our infrastructure with good performance and stability.

\subsection{CMSDbBrowser Monitoring functionalities}

Monitoring and fast error detection in real time systems is a very challenging task, since spotting a faulty situation requires strict timing constraints and a fast reaction, in order to recover the system and to put it into a correct state. Here we monitor the status of job consumption by online and offline processes. If any jobs fail we get notification which help experts to take action quickly.

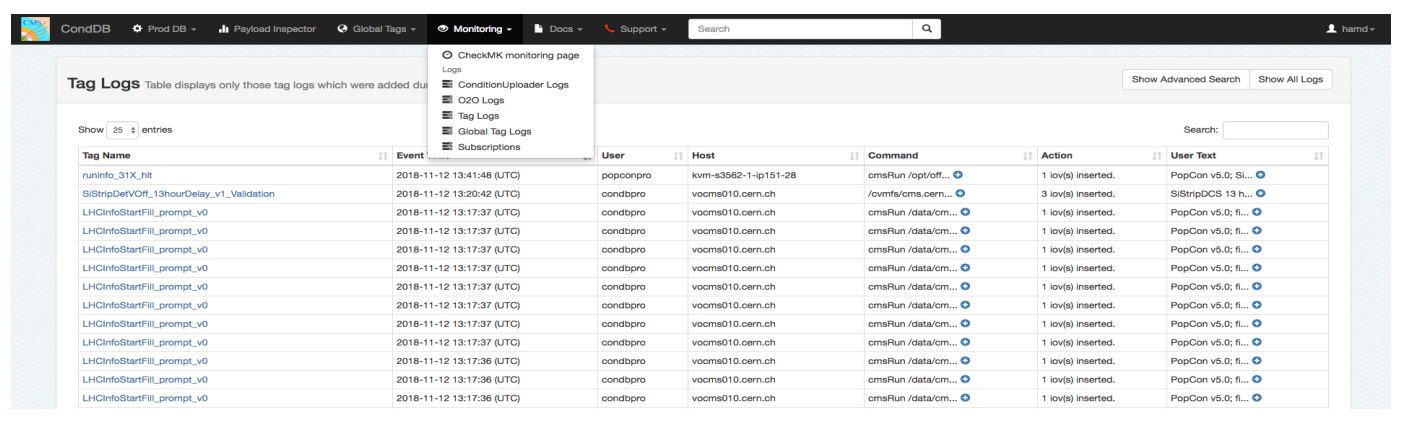

Figure 2. CmsDbBrowser Monitoring Service

As shown in Figure 2, Monitoring section in the cmsDbBrowser displays various different log pages:

- ConditionUploader logs, showing information about the ConditionUploader service;

- $\mathrm{O} 2 \mathrm{O} \operatorname{logs}$, displaying $\mathrm{O} 2 \mathrm{O}$ job details and job run information;

- Tag logs, showing recent changes to Tags;

- Global Tag logs, showing recent changes to Global Tags.

\section{CMS Conditions Browser As a Service}

In 2017 we incorporated a few new tools to our Condition Browser service which has turned out very useful for detector expert to easily inspect their data and get notified when changes are made to there data. 


\subsection{Subscription Service}

The cmsDbBrowser service provides users with a customizable way to receive updates about modification and creation of data in the conditions database. This service allows the user to subscribe to events in the Tag Log and Global Tag Log. The server will periodically fetch the latest events from the logs, match them to subscriptions, and for each subscriber generate a personalized activity digest which will be sent by email.

- Subscription Entities: A subscription also has a set of subscribed entities. These will determine which events go into the activity digest email. Subscribed entities may be provided when the subscription is created, but can also be added or removed later by selecting the given subscription.

- Subscription Emails: Activity digest emails generated by this service works in such a way that each email will always contain all relevant events that has happened since the last automatic email was sent. An additional feature allows you to request an instant email from the browser page.

\subsection{Payload Inspector}

The Payload Inspector was designed in order to allow detector experts to easily inspect, monitor and share the alignment and calibration constants stored in the CMS conditions database by generating interactive plots from user selected data. Condition proponents can choose and develop the display format depending on their needs in the CMS software framework(CMSSW) as Payload Inspector plugins in the CondCore package. The plots are developed in $\mathrm{C}++$ by the experts of each system following a schema of templated $\mathrm{C}++$ classes, and thus the browser can discover the monitoring plots by discovering dynamically from CMS Software (CMSSW) releases. The tool consists of two separate layers:

- Deserialisation layer: Dedicated plugins in the CMSSW load and deserialise payloads from the conditions database, extract the relevant information, and compute the quantities to be displayed; such quantities are packaged in a json format which holds all the information necessary for rendering of the plots.

- Visualisation layer: Receives the information necessary for rendering of the plots and generates interactive views for the actual inspection of conditions. This layer is integrated in the cmsDbBrowser web-based application, which is the main entry point accessible for all CMS members to browse and manipulate conditions data and metadata.

As shown in Figure 3, this design allows users to select the data they want to visualize. The CmsDbBrowser fetches the user selected data and displays it in the form of a plot dynamically. A user can easily inspect and monitor selected calibration measurements and see the time evolution of the data.

\section{Conclusions}

The CMS conditions database browser service was created to streamline and structure the management of alignment and calibration sets which are fundamental to the CMS detector operated at the CERN Large Hadron Collider (LHC). A set of tools has been provided to the production managers for the tag and global tag management and data transfer. The service was designed to provide a single entry point for any CMS member to query the conditions database and give an answer to all conditions related needs. It meets the requirements of the CMS collaborators and saves a lot of valuable time for the experts and managers. This 

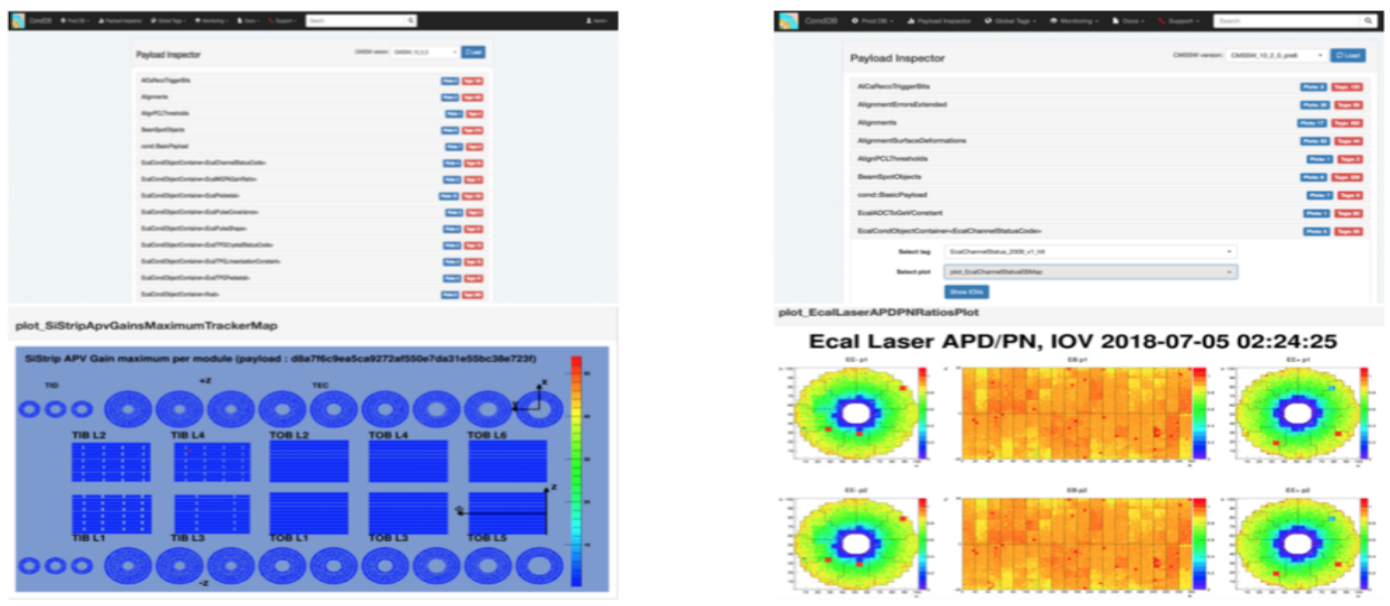

Figure 3. Data Visualisation using Payload Inspector

application has been proven vital in catching the problems and there have been incidents which we have solved because of quick notifications from this service which basically results in maintaining the good quality of data for physics analysis.

\section{References}

[1] CMS COLLABORATION, The CMS experiment at the CERN LHC, JINST 3 S08004, doi: https://iopscience.iop.org/article/10.1088/1748-0221/3/08/S08004 (2008)

[2] CMS COLLABORATION, CMS High Level Trigger, CERN/LHCC 2007-021 doi: https://iopscience.iop.org/article/10.1088/1742-6596/664/8/082044(2007)

[3] S.Di Guida, G.Govi, M.Ojeda and A.Pfeiffer, Multi-threaded Object Streaming , J. Phys.: Conf. Ser. 664042044 (2015), doi: https://iopscience.iop.org/article/10.1088/17426596/664/4/042044 (2015)

[4] S.Di Guida, G.Govi, M.Ojeda and A.Pfeiffer, The CMS Condition Database system, J. Phys.: Conf. Ser. 664042024 (2015), doi: https://iopscience.iop.org/article/10.1088/17426596/664/4/042024 (2015)

[5] A.Mecionis, Salvatore Di Guida, Giovanni Franzoni, Marco Musich, Gianluca Cerminara, Andreas Pfeiffer and Giacomo Govi, A Web-based application for the collection, management and release of Alignment and Calibration configurations used in data processing at the Compact Muon Solenoid experiment, Journal of Physics: Conf. Series 898 (2017) 032034 doi: https://iopscience.iop.org/article/10.1088/1742-6596/898/3/032034 (2017) 